\title{
Systemic Hypertension and Microalbuminuria
}

\author{
Ana Maria Nunes de Faria Stamm, Gisele Meinerz, Jacqueline Consuelo da Silva \\ Hospital Universitário Prof. Dr. Polydoro Ernani São Thiago - Universidade Federal de Santa Catarina - Florianópolis, SC - Brazil
}

\author{
Summary \\ Objetivo: To identify the prevalence of microalbuminuria and target organ lesions and their association in a hypertensive \\ population undergoing treatment.
}

Methods: This observational, descriptive and cross-sectional study was conducted between April and August 2006, and included 153 hypertensive patients undergoing treatment at the Internal Medicine and Cardiology Outpatient Clinics at a University Hospital in the Southern Region of Brazil.

Results: This observational, descriptive and cross-sectional study was conducted between April and August 2006, and included 153 hypertensive patients undergoing treatment at the Internal Medicine and Cardiology Outpatient Clinics at a University Hospital in the Southern Region of Brazil.

Conclusion: The prevalence of microalbuminuria in the study population was $13.7 \%$ and that of target organ lesions was $48.4 \%$; a statistically significant association was found. Microalbuminuria is also associated with cardiac lesions, including in the geriatric population. (Arq Bras Cardiol 2007;89(6):376-381)

Key words: Hypertension; albuminuria; target organs; risk factors.

\section{Introduction}

Systemic hypertension $(\mathrm{SH})$ is currently defined as systolic blood pressure (SBP) greater than or equal to $140 \mathrm{mmHg}$ and/ or diastolic blood pressure (DBP) greater than or equal to 90 $\mathrm{mmHg}$ in adults 18 years of age or older ${ }^{1-4}$. Throughout time this classification has been modified, as new studies evaluate patient evolution in accordance with blood pressure levels.

Great importance has been given to microalbuminuria as a prognostic marker of cardiovascular and/or renal risk in diabetics 5,6 , hypertensives ${ }^{6-10}$ and the population as a whole ${ }^{11-13}$. Studies have demonstrated an ongoing association of microalbuminuria with cardiovascular events and kidney lesions, that is, the higher the urinary albumin excretion the greater the risk to develop these conditions ${ }^{14}$.

Microalbuminuria is defined as elevated urinary albumin excretion, exceeding normal levels without reaching the minimum levels that can be detected by routine laboratory methods, that is, an albumin excretion between 30 and 300 milligrams in 24 hours $^{5,15-17}$. These cut-off points were defined based on studies that show the risk curve to develop nephropathy in diabetic patients ${ }^{18}$, and has been questioned by some authors ${ }^{12,19-21}$.

The mechanism that causes increased urinary albumin excretion is not well defined, and the reasons why this excretion is associated with increased cardiovascular risk have

Mailing address: Ana Maria Nunes de Faria Stamm •

Rua: Germano Wendhausen, 32/101 - Edifício Domus - Centro

88015-460 - Florianópolis, SC - Brazil

E-mail: stamm@ativanet.com.br

Manuscript received March 20, 2007; revised manuscript received May 25, 2007; accepted June 14, 2007. not yet been established. The prevailing explanation is that microalbuminuria represents a generalized dysfunction of vascular endothelium, increasing permeability and causing the leakage of albumin through the glomerular membrane ${ }^{17,18,22}$.

Even though there are disparities between the various studies, the prevalence of microalbuminuria generally ranges between $20 \%$ and $30 \%$ in untreated hypertensive patients and is as high as $25 \%$ in patients undergoing anti-hypertensive treatment ${ }^{7,8,10,23}$.

Owing to the importance of the risk factors when assessing $\mathrm{SH}$ patients and the prognostic value attributed to microalbuminuria, the objective of the present study is to identify the prevalence of microalbuminuria and the association between this risk marker and target organ lesions, in a population of hypertensive patients undergoing antihypertensive treatment.

\section{Method}

An observational, descriptive and cross-sectional study was conducted at the Internal Medicine Outpatient Healthcare Center and the Cardiology Outpatient Clinic at a University Hospital in the Southern Region of Brazil, between April and August 2006.

The medical charts of the patients were reviewed in order to select $\mathrm{SH}$ patients that had not been diagnosed with diabetes mellitus, and when they arrived for their previously scheduled consultations at the abovementioned outpatient clinics, they were invited for an interview. Therefore, from the 201 patients that agreed to participate in the study, 153 met the inclusion criteria, comprising the final sample. 
Patients with essential $\mathrm{SH}$, undergoing anti-hypertensive treatment, from both genders, 18 years of age or older, who agreed to participate in the study and supplied an isolated early morning urine sample were included in the study. Patients with clinical and/or laboratory evidence of secondary $\mathrm{SH}$, a diagnosis of diabetes mellitus and chronic kidney disease were excluded from the study. Patients who presented a partial urine test with signs of urinary tract infection or proteinuria were also excluded.

The following variables were evaluated: age, gender, race, body mass index (BMI), smoking, sedentary lifestyle, presence of target organ lesions and cardiovascular disease, lipid profile, blood pressure levels, SH duration, and presence of microalbuminuria. The patients were divided into two groups based on age: geriatrics (60 years or older) and non-geriatrics (younger than 60 years).

A ratio between the concentration of albumin and urinary creatinine within an interval of 30 to 300 milligrams of albumin per gram of creatinine was considered positive for microalbuminuria. The albuminuria assay was conducted using the nephelometry technique (Behring Nephelometer 100, Dade Behring $($ ) and urinary creatinine was measured using the Jaffe technique. After proper unit conversions, the albumin/creatinine ratio was obtained, except when the urinary albumin concentration was less than 12mg/l (lowest equipment detection limit).

This study was approved by the University's Human Research Ethics Committee. All the patients signed a free and informed consent form.

The data were analyzed using the program Epi Info ${ }^{\circledR}$ v.6.04. The chi-square test $\left(\chi^{2}\right)$ was used to compare variable prevalence between the groups, using a confidence interval of $95 \%(p<0.05)$. The F-statistics variance analysis was used to compare the mean values between the groups, with the same confidence interval.

\section{Results}

Between April and August 2006, 201 patients from the Internal Medicine Outpatient Healthcare Center and the Cardiology Outpatient Clinic at a University Hospital were interviewed; 153 met the inclusion criteria. From these, 21 presented microalbuminuria (13.7\%).

The normoalbuminuria and microalbuminuria groups were similar, with a predominance of females [91/132 (68.9\%) versus $12 / 21(57.1 \%)]$, white race $[116 / 132$ (87.8\%) versus $18 / 21(85.7 \%)]$ and a mean age of $60.7 \pm 13$ years for the patients with normoalbuminuria and $63.9 \pm 12.7$ for those with microalbuminuria (Table 1).

In relation to clinical characteristics, smoking [29.5\% $(39 / 132)$ versus $28.6 \%(6 / 21)]$, sedentary lifestyle [59.1\% $(78 / 132)$ versus $47.6 \%(10 / 21)]$ and obesity [31.8\% $(42 / 132)$ versus $23.8 \%(5 / 21)]$ were more prevalent in the normoalbuminuria group, while dyslipidemia was more prevalent in the microalbuminuria group $[52.4 \%(11 / 21)$ versus $34.8 \%$ (46/132)] (Table 2). In relation to lipid levels, a statistically significant difference was only found for HDL cholesterol $(46.0 \pm 12.9 \mathrm{mg} / \mathrm{dl}$ for those with normoalbuminuria versus $39.7 \pm 10.2 \mathrm{mg} / \mathrm{dl}$ for those with microalbuminuria; $\mathrm{p}=0.03$ )

The two groups were similar in relation to SBP $(143.3 \pm 18.3$ $\mathrm{mmHg}$ versus $142.5 \pm 21.5 \mathrm{mmHg}), \mathrm{DBP}(88.0 \pm 12.0 \mathrm{mmHg}$ versus $86.2 \pm 10.7 \mathrm{mmHg}$ ) and $\mathrm{SH}$ duration $(116.3 \pm 104.4$ months versus $114.9 \pm 139.6$ months) (Table 2).

The prevalence of at least one target organ lesion was $48.4 \%(74 / 153)$ in the general population, and was greater in the microalbuminuria group [76.2\% (16/21) versus $43.9 \%$ (58/132) in the normoalbuminuria patients], which was statistically significant $(p=0.006)$ (Table 3$)$.

The most common lesions presented by the study patients were cardiac [41.8\% (64/153)] particularly angina and/or acute myocardial infarction (AMI) [41/153 (26.8\%)] and left ventricular hypertrophy (LVH) [21/153 (13.7\%)] followed by congestive heart failure (CHF) [17/153 (11.1\%)]. Encephalitic stroke (ES) or transient ischemic attack (TIA) [10/153 (6.5\%)]; peripheral arterial disease [10/153 (6.5\%)] and hypertensive retinopathy [2/153 (1.3\%)] were less prevalent (Table 3).

A statistically significant association was found between cardiac lesions and microalbuminuria $(p=0.003)$ which were presented by $71.4 \%$ (15/21) of the patients, while only $37.1 \%$ $(49 / 132)$ of the normoalbuminuria patients presented this type of lesion (Table 3).

Half of the study population was comprised by the geriatric group $[51 \%(78 / 153)]$ and the other half by the non-geriatric group [49\% (75/153)] with a similar microalbuminuria prevalence in both groups $[15.4 \%(12 / 78)$ versus $12.0 \%$ (9/75)] (Table 4).

There was a statistically significant association $(p=0.006)$ between the presence of microalbuminuria and cardiac lesions in the geriatric group; however, this was not found in the non-geriatric group (Table 5). Using logistic regression analysis, we observed that the microalbuminuria, and not age, has the greatest association with cardiac lesions $(p=0.005)$ (Table 6).

\section{Discussion}

Systemic hypertension and its complications are responsible for a large portion of the hospital admissions in Brazil, representing a very high socioeconomic cost. During 2003, cardiovascular diseases were responsible for $27.4 \%$ of the deaths in the country, with a direct relation between cardiovascular mortality and blood pressure levels ${ }^{2}$.

Considering that roughly $20 \%$ to $40 \%$ of the Brazilian population have blood pressure levels greater than or equal to 140 per $90 \mathrm{mmHG}^{1,2}$, the importance of this relationship and the necessity to study factors related to it is recognized.

Microalbuminuria was initially defined as a urinary albumin excretion value that would be related to a greater risk to develop nephropathy in diabetic patients ${ }^{18}$. Studies conducted later demonstrated that microalbuminuria is also associated with worse cardiovascular outcomes, even in the general population ${ }^{18,24}$. Although the mechanism is still not clear, it is believed to be a renal indication of a generalized disorder of vascular endothelium with permeability alterations ${ }^{17,18,22}$. 


\section{Original Article}

\begin{tabular}{|c|c|c|c|c|}
\hline & Group NA $(n=132)$ & Group MA $(n=21)$ & Total $(n=153)$ & $\mathbf{p}^{*}$ \\
\hline Female Gender (\%) & $91(68.9)$ & $12(57.1)$ & $103(67.3)$ & 0.28 \\
\hline White (\%) & $116(87.8)$ & 18 (85.7) & $134(87.6)$ & 0.72 \\
\hline Age $\pm S D$ & $60.7 \pm 13.0$ & $63.9 \pm 12.7$ & $61.1 \pm 13$ & 0.29 \\
\hline
\end{tabular}

\begin{tabular}{|c|c|c|c|c|}
\hline & Group NA $(n=132)$ & Group MA $(n=21)$ & Total $(n=153)$ & $\mathbf{p}^{*}$ \\
\hline Smoking (\%) & $39(29.5)$ & $6(28.6)$ & $45(29.4)$ & 0.92 \\
\hline Sedentary lifestyle (\%) & $78(59.1)$ & $10(47.6)$ & $88(57.5)$ & 0.32 \\
\hline Obesity (\%) & $42(31.8)$ & $5(23.8)$ & $47(30.7)$ & 0.46 \\
\hline Dyslipidemia (\%) & $46(34.8)$ & $11(52.4)$ & $57(37.2)$ & 0.12 \\
\hline $\mathrm{SBP} \pm \mathrm{SD}$ & $143.3 \pm 18.3$ & $142.5 \pm 21.5$ & $143.2 \pm 18.7$ & 0.85 \\
\hline $\mathrm{DBP} \pm \mathrm{SD}$ & $88.0 \pm 12.0$ & $86.2 \pm 10.7$ & $87.7 \pm 11.8$ & 0.52 \\
\hline SH duration, months \pm SD & $116.3 \pm 104.4$ & $114.9 \pm 139.6$ & $116.1 \pm 109.3$ & 0.95 \\
\hline
\end{tabular}

Since the relation between microalbuminuria and a worse cardiovascular prognosis has been established, various authors have tried to determine the variables associated with it, in order to define a profile of high risk patients and the possible strategies to prevent or diminish the progression to kidney disease and cardiovascular events.

First, we should remember that there are individual variations in urinary albumin excretion during the day and on different days. Therefore, the diagnosis of microalbuminuria should only be established when two of three samples of first morning urine present an altered albumin/creatinine ratio $^{16,24,25}$.

Among the factors that can cause transitory increases of urinary albumin excretion are overtiring physical exertion, urinary tract infection, decompensated heart failure, acute febrile diseases and vaginal secretions ${ }^{24-26}$. Blood pressure elevations during pregnancy or the development of gestational diabetes also cause significant increases in albuminuria ${ }^{26}$. These factors should be considered in the evaluation of the test results.

Some studies demonstrate associations between microalbuminuria and gender, race, age, blood pressure levels, cholesterol fractions, smoking and $\mathrm{BMI}^{19,27}$; however, in the majority of the studies there is a great deal of controversy in regard to study variables.

For example, Agrawal et $\mathrm{al}^{8}$ demonstrated an association with age, male gender, $\mathrm{SH}$ duration, treatment duration and blood pressure levels, whereas Nakamura and associates ${ }^{10}$, Clausen et $\mathrm{al}^{11}$ and Redon et $\mathrm{al}^{28}$ did not observe this relationship.
Three other studies with untreated hypertensive patients also present conflicting results. Palatini et al ${ }^{29}$ did not observe a relation with smoking, physical activity, BMI, age, family history of early onset cardiovascular disease or SH duration, whereas Leoncini et $\mathrm{al}^{30}$ found an association with age, BMI, smoking, HDL cholesterol, triglycerides and blood pressure levels. Pontremoli et $\mathrm{al}^{31}$ found a relation with male gender, SBP, DBP, HDL cholesterol and BMI, but not with a family history of early onset cardiovascular disease, smoking or a sedentary lifestyle.

In the present study, the groups with and without microalbuminuria were similar, only presenting a statistically significant difference for HDL cholesterol levels, and the microalbuminuria patients presented values below $40 \mathrm{mg} / \mathrm{dl}$ more often.

There is also a great deal of variation in regard to the prevalence of microalbuminuria. In the untreated hypertensive population, the prevalence is considered to be between $20 \%$ and $30 \%$, and in the treated hypertensive population it is as high as $25 \%^{22,23}$, even though some authors accept values between $7 \%$ and $40 \%{ }^{15,25}$. Explanations for this variation could be different urinary albumin measurement methods, urinary collection errors (when conducted over a period of time) or study group variations in relation to age, ethnic groups or comorbidities $^{18}$.

In cross-sectional studies, Jensen et $\mathrm{a}^{32}$ found a prevalence of $4.7 \%$ in hypertensive patients undergoing treatment, whereas Palatini et $\mathrm{al}^{29}$, Pontremoli et $\mathrm{al}^{31}$ and Leoncini et $\mathrm{al}^{30}$ found, respectively, $6.1 \%, 6.7 \%$ and $9 \%$ in untreated hypertensive patients. 
Table 3 - Relationship between microalbuminuria and target organ lesions in hypertensive patients undergoing treatment

\begin{tabular}{lcccc} 
& Group NA $(\mathbf{n}=\mathbf{1 3 2})$ & Group MA (n= 21) & Total $(\mathbf{n}=\mathbf{1 5 3})$ & $\mathbf{p}^{*}$ \\
\hline TOL present (\%) & $58(43.9)$ & $16(76.2)$ & $74(48.4)$ & 0.006 \\
Cardiac (\%) & $49(37.1)$ & $15(71.4)$ & $64(41.8)$ & 0.003 \\
ES/TIA (\%) & $9(6.8)$ & $1(4.8)$ & $10(6.5)$ & 1.00 \\
PAD (\%) & $8(6.1)$ & $2(9.5)$ & $10(6.5)$ & 0.62 \\
Retinopathy (\%) & $2(1.5)$ & 0 & $2(1.3)$ & 0.81 \\
\hline
\end{tabular}

NA - normoalbuminuria; MA - microalbuminuria; TOL - target organ lesion; Cardiac - left ventricular hypertrophy, angina and/or acute myocardial infarction, congestive heart failure; ES - encephalitic stroke; TIA - transient ischemic attack; PAD - peripheral arterial disease.

*: analysis using the chi-square test

\begin{tabular}{|lccccc}
\hline \multicolumn{5}{|c}{ Table 4 - Relationship between microalbuminuria and age group in hypertensive patients undergoing treatment } \\
\hline & Geriatrics $(\mathbf{n}=\mathbf{7 8})$ & Non-geriatrics $(\mathbf{n}=\mathbf{7 5})$ & Total $(\mathbf{n}=\mathbf{1 5 3})$ & $\mathbf{p}^{*}$ \\
\hline Group NA (\%) & $66(84.6)$ & $66(88.0)$ & $132(86.3)$ & 0.54 \\
\hline Group MA (\%) & $12(15.4)$ & $9(12.0)$ & $21(13.7)$ \\
\hline
\end{tabular}

NA - normoalbuminúria; MA - microalbuminúria; *: teste de qui-quadrado.

\begin{tabular}{|lcccc}
\hline \multicolumn{2}{|c}{ Table $\mathbf{5}$ - Relationship between microalbuminuria, cardiac lesions and age group in hypertensive patients undergoing treatment } \\
\hline & \multicolumn{2}{c}{ Geriatrics * } & \multicolumn{3}{c}{ Non-geriatrics } \\
\hline \\
\hline Cardiac lesion + & Group NA & Group MA & Group NA & Group MA \\
Cardiac lesion - & $27(40.9)$ & $10(83.3)$ & $22(33.3)$ & $5(55.6)$ \\
\hline
\end{tabular}

NA - normoalbuminuria; MA - microalbuminuria; Cardiac lesion - left ventricular hypertrophy, angina and/or acute myocardial infarction, congestive heart failure; *: $\chi^{2}=7.33 ; p=0.006$.; The numbers in the brackets indicate the percentages.

\begin{tabular}{|c|c|c|c|c|c|c|c|}
\hline & Coefficient & Standard Error & Wald & p & OR & $\begin{array}{c}95 \% \mathrm{Cl} \\
\text { minimum }\end{array}$ & $\begin{array}{c}95 \% \mathrm{Cl} \\
\text { maximum }\end{array}$ \\
\hline Age $\geq 60$ years & 0.412 & 0.347 & 1.412 & 0.235 & 1.510 & 0.806 & 3.056 \\
\hline Microalbuminuria & 1.481 & 0.529 & 7.851 & 0.005 & 4.398 & 1.511 & 11.519 \\
\hline Constant & -0.672 & 0.299 & 5.055 & 0.025 & 0.511 & & \\
\hline
\end{tabular}

On the other hand, Agewall et al $^{7}$ observed microalbuminuria in $25 \%$ of their patients receiving antihypertensive treatment, and Agrawal et $\mathrm{al}^{8}$ found a prevalence close to $30 \%$, in a study involving treated and untreated hypertensive patients. In a prospective study, Redon et $\mathrm{al}^{28}$ followed a group of hypertensive patients for roughly 2.7 years and observed the progression to microalbuminuria in $11.7 \%(22 / 187)$.

In our study, the microalbuminuria prevalence was $13.7 \%$, a result within the expected range for the type of population studied.

Various studies have demonstrated a positive and independent association between microalbuminuria and cardiovascular disease in both hypertensive patients ${ }^{7,8}$ and the general population ${ }^{12,13}$.

In 1997, Jensen et $\mathrm{a}^{32}$ published a study demonstrating that urinary albumin excretion (and not the presence of microalbuminuria) was related to cardiovascular disease, and that the highest blood pressure levels were found in the patients with the highest urinary albumin excretion. In 2000, the same authors ${ }^{9}$ published a study that followed hypertensive patients for 10 years and observed the development of ischemic coronary disease in $28 \%$ of the patients that had microalbuminuria at the start of the study, whereas this rate was only $8 \%$ in the patients with normoalbuminuria. 


\section{Original Article}

In the present study, a statistically significant association was found between target organ lesions and microalbuminuria $(p=0.006)$; these lesions were found in approximately $3 / 4$ of the patients in this group $(76.2 \%$ versus $43.9 \%$ in the normoalbuminuria group). Cardiac lesions had the highest prevalence and maintained a significant association with microalbuminuria $(p=0.003)$, with a frequency of $71.4 \%$ of the patients in this group.

Nakamura et $\mathrm{al}^{10}$ followed hypertensive patients over the age of 65 for eight years, comprising 111 normoalbuminuria patients and 33 microalbuminuria patients. The incidence of cardiovascular disease in the microalbuminuria group was higher (18\% versus $7 \%$ ) and the urinary albumin excretion values were related to the incidence of cardiovascular disease.

In the group of geriatric microalbuminuria patients, the majority $(83.3 \%)$ presented some type of cardiac lesion. In the individuals under age 60, bivariate analysis did not reveal an association between microalbuminuria and cardiac lesions, but the logistic regression analysis demonstrated that microalbuminuria and not age group, was the variable that determined the presence of cardiac lesions.

Since this was a cross-sectional study, we could not establish a causal relationship between microalbuminuria and target organ lesions or, more specifically, cardiac lesions; however we can assume that there is an association between these two conditions, which has also been indicated by the various studies on the subject.

The sample is another point to be considered. Since the population was selected on a convenience basis, the results cannot be extrapolated for the general population; however it can be determined that the results are in agreement with medical literature in the area.

The results of the present study summarize the findings in other studies, and could encourage the development of new studies to further our comprehension on the association

\section{References}

1. IV Diretrizes Brasileiras de Hipertensão Arterial. Arq Bras Cardiol. 2004; 82 (supl 4): 7-22.

2. V Diretrizes Brasileiras de Hipertensão Arterial. [Acesso em 2006 maio 21]. Disponível em <http://www.diabetes.org.br >..

3. The Seventh Report of the Joint National Committee on Prevention, Detection, Evaluation and Treatment of High Blood Pressure. The JNC 7 Report. JAMA. 2003; 289 (19): 2560-71.

4. 2003 European Society of Hypertension-European Society of Cardiology guidelines for the management of arterial hypertension. J Hypertens . 2003; 21(6): 1011-53.

5. Sukhija R, Aronow WS, Kakar P, Garza L, Sachdeva R, Sinha A, et al. Relation of microalbuminuria and coronary artery disease in patients with and without diabetes mellitus. Am J Cardiol. 2006; 98 (3): 279-81.

6. Weir MR, Blantz RC. The clinical utilization of albuminuria as a surrogate measure of cardiovascular disease burden and risk for events: are we there yet? Curr Opin Nephrol Hypertens. 2005; 14 (1): 39-41.

7. Agewall S, Persson B, Samuelsson O, Ljungman S, Herlitz H, Fagerberg B. Microalbuminuria in treated hypertensive men at high risk of coronary disease. The Risk Factor Intervention Study Group. J Hypertens. 1993; 11 (4): 461-9. between microalbuminuria and systemic hypertension.

Microalbuminuria is an important auxiliary tool in the assessment of hypertensive patients, and should be studied and investigated more thoroughly in medical practice. Microalbuminuria isn't the cause of cardiovascular disease, but its presence helps to identify individuals at high risk to develop it, indicating that they should be followed more closely and receive aggressive treatment for other concomitant risk factors ${ }^{25}$.

\section{Conclusion}

For the hypertensive population treated at the Internal Medicine Outpatient Healthcare Center and the Cardiology Outpatient Clinic at a University Hospital in the Southern Region of Brazil between April and August 2006, in regard to the proposed objectives, we can conclude:

1. The prevalence of microalbuminuria is $13.7 \%$ and that of target organ lesions is $48.4 \%$;

2. There is a statistically significant association between microalbuminuria and target organ lesions. This association holds true in relation to cardiac lesions for the entire study population, including the geriatric group.

\section{Potential Conflict of Interest}

No potential conflict of interest relevant to this article was reported.

\section{Sources of Funding}

There were no external funding sources for this study.

\section{Study Association}

This study is not associated with any graduation program.
8. Agrawal B, Berger A, Wolf K, Luft FC. Microalbuminuria screening by reagent strip predicts cardiovascular risk in hypertension. J Hypertens. 1996; 14 (2): 223-8.

9. Jensen JS, Feldt-Rasmussen B, Strandgaard S, Schroll M, Borch-Johnsen K. Arterial hypertension, microalbuminuria, and risk of ischemic heart disease. Hypertension. 2000; 35 (4): 898-903.

10. Nakamura S, Kawano Y, Inenaga T, Nakahama H, Horio T, Sasaki O, et al. Microalbuminuria and cardiovascular events in elderly hypertensive patients without previous cardiovascular complications. Hypertens Res. 2003; 26 (8): 603-8.

11. Clausen P, Jensen JS, Borch-Johnsen K, Jensen G, Feldt-Rasmussen B. Ambulatory blood pressure and urinary albumin excretion in clinically healthy subjects. Hypertension. 1998; 32 (1): 71-7.

12. Klausen K, Borch-Johnsen K, Feldt-Rasmussen B, Jensen G, Clausen P, Scharling $\mathrm{H}$, et al. Very low levels of microalbuminuria are associated with increased risk of coronary heart disease and death independently of renal function, hypertension, and diabetes. Circulation. 2004; 110 (1): 32-5.

13. Yuyun MF, Khaw KT, Luben R, Welch A, Bingham S, Day NE, et al. Microalbuminuria, cardiovascular risk factors and cardiovascular morbidity in a British population: the EPIC-Norfolk population-based study. Eur J Cardiovasc Prev Rehabil. 2004; 11 (3): 207-13. 
14. Ljungman S, Wikstrand J, Hartford M, Berglund G. Urinary albumin excretion -- a predictor of risk of cardiovascular disease: a prospective 10-year follow-up of middle-aged nondiabetic normal and hypertensive men. Am J Hypertens. 1996; 9 (8): 770-8.

15. Campese VM, Bianchi S, Bigazzi R. Is microalbuminuria a predictor of cardiovascular and renal disease in patients with essential hypertension? Curr Opin Nephrol Hypertens. 2000; 9 (2): 143-7.

16. Tagle R, Acevedo M, Vidt DG. Microalbuminuria: is it a valid predictor of cardiovascular risk? Cleve Clin J Med. 2003; 70 (3): 255-61.

17. Kopyt NP. Slowing progression along the renal disease continuum. J Am Osteopath Assoc. 2005; 105 (4): 207-15.

18. Yuyun MF, Adler Al, Wareham NJ. What is the evidence that microalbuminuria is a predictor of cardiovascular disease events? Curr Opin Nephrol Hypertens. 2005; 14 (3): 271-6.

19. Jacobs Jr DR, Murtaugh MA, Steffes M, YuX, Roseman J, Goetz FC. Gender- and race-specific determination of albumin excretion rate using albumin-to-creatinine ratio in single, untimed urine specimens: the Coronary Artery Risk Development in Young Adults Study. Am J Epidemiol. 2002; 155 (12): 1114-9.

20. Yuyun MF, Khaw KT, Luben R, Welch A, Bingham S, Day NE, et al. A prospective study of microalbuminuria and incident coronary heart disease and its prognostic significance in a British population: the EPIC-Norfolk study. Am J Epidemiol. 2004; 159 (3): 284-93.

21. Ratto E, Leoncini G, Viazzi F, Vaccaro V, Parodi A, Falqui V, et al. Microalbuminuria and cardiovascular risk assessment in primary hypertension: should threshold levels be revised? Am J Hypertens. 2006; 19 (7): 728-34; discussion 735-6.

22. Ruilope LM. Kidney dysfunction: a sensitive predictor of cardiovascular risk. Am J Hypertens. 2001; 14 (6 Pt 2): 213S-217S,

23. Ruilope LM, van Veldhuisen DJ, Ritz E, Luscher TF. Renal function: the Cinderella of cardiovascular risk profile. J Am Coll Cardiol. 2001; 38 (7): 1782-7.
24. Redon J. Measurement of microalbuminuria -- what the nephrologist should know. Nephrol Dial Transplant. 2006; 21 (3): 573-6.

25. Keane WF, Eknoyan G. Proteinuria, albuminuria, risk, assessment, detection elimination (PARADE): a position paper of the National Kidney Foundation. Am J Kidney Dis. 1999; 33 (5): 1004-10.

26. Mogensen CE, Vestbo E, Poulsen PL, Christiansen C, Damsgaard EM, Eiskjaer $\mathrm{H}$, et al. Microalbuminuria and potential confounders: a review and some observations on variability of urinary albumin excretion. Diabetes Care. 1995; 18 (4): 572-81.

27. Cirillo M, Senigalliesi L, Laurenzi M, Alfieri R, Stamler J, Stamler R, et al. Microalbuminuria in nondiabetic adults: relation of blood pressure, body mass index, plasma cholesterol levels, and smoking: The Gubbio Population Study. Arch Intern Méd. 1998; 158 (17): 1933-9.

28. Redon J, Rovira E, Miralles A, Julve R, Pascual JM. Factors related to the occurrence of microalbuminuria during antihypertensive treatment in essential hypertension. Hypertension. 2002; 39 (3): 794-8.

29. Palatini P, Graniero GR, Mormino P, Mattarei M, Sanzuol F, Cignacco GB, et al. Prevalence and clinical correlates of microalbuminuria in stage hypertension: results from the Hypertension and Ambulatory Recording Venetia Study (HARVEST Study). Am J Hypertens. 1996; 9 (4 Pt 1): 334-41.

30. Leoncini G, Viazzi F, Parodi D, Vettoretti S, Ratto E, Ravera M, et al. Mild renal dysfunction and subclinical cardiovascular damage in primary hypertension. Hypertension. 2003; 42 (1): 14-8.

31. Pontremoli R, Sofia A, Ravera M, Nicolella C, Viazzi F, Tirotta A, et al Prevalence and clinical correlates of microalbuminuria in essential hypertension: the MAGIC Study. Microalbuminuria: a Genoa Investigation on Complications. Hypertension. 1997; 30 (5): 1135-43.

32. Jensen JS, Feldt-Rasmussen B, Borch-Johnsen K, Clausen P, Appleyard M Jensen $G$. Microalbuminuria and its relation to cardiovascular disease and risk factors: a population-based study of 1254 hypertensive individuals. J Hum Hypertens. 1997; 11 (11): 727-32. 